\title{
Derivation and validation a risk model for acute kidney injury and subsequent adverse events after cardiac surgery: a multicenter cohort study
}

\section{Hang Zhang}

Nanjing First Hospital, Nanjing Medical University

Min Yu

Shanghai General Hospital

Rui Wang

Nanjing First Hospital

Rui Fan

Nanjing First Hospital

Ke Zhang

Changzhou Second People's Hospital

Wen Chen

Nanjing First Hospital

Xin Chen ( $\nabla$ stevecx@njmu.edu.cn )

Nanjing First Hospital, Nanjing medical university https://orcid.org/0000-0002-7786-1701

Research article

Keywords: Acute kidney injury, Cardiac surgery, Prediction model, Nomogram

Posted Date: October 18th, 2021

DOl: https://doi.org/10.21203/rs.3.rs-962782/v1

License: (c) (i) This work is licensed under a Creative Commons Attribution 4.0 International License.

Read Full License 


\section{Abstract \\ Background}

Acute Kidney Injury, a frequent complication in patients undergoing cardiac surgery, is associated with high mortality and poor quality of life. We aimed to establish a risk model for acute kidney injury and subsequent adverse events in Chinese cardiac patients.

\section{Methods}

This study included 11740 patients who had cardiac surgery at 14 institutions in China. Patients were randomly assigned to a derivation cohort $(n=8197)$ or a validation cohort $(n=3543)$. Variables ascertained during hospitalization were screened using least absolute shrinkage and selection operator and logistic regression to construct a nomogram model. Model performance was evaluated using Cstatistic, calibration curve, and Brier score. The nomogram was further compared with the five conventional models: Mehta score, Ng score, AKICS score, SRI score, and Cleveland Clinic score. Acute kidney injury was defined according to the Kidney Disease Improving Global Outcomes criteria. Subsequent adverse events included mid-term outcomes: death from all causes and major adverse kidney events (defined as composite outcome of death from renal failure, dialysis, and advanced chronic kidney disease).

\section{Results}

Acute kidney injury occurred in 3237 (27.6\%) patients. The model included 12 predictors. The nomogram achieved a C-statistic of 0.825 and 0.804 in the derivation and validation cohorts, respectively, and had well-fitted calibration curves. The model performance of the nomogram was better than other five conventional models. After risk stratification, moderate-risk or high-risk groups were associated with significantly higher rates of death from all causes and major adverse kidney events compared with lowrisk group during 7-year follow-up.

\section{Conclusions}

The nomogram provided an effective tool for predicting acute kidney injury and evaluating its subsequent adverse events after cardiac surgery.

\section{Introduction}

Cardiac surgery is more and more frequent in a growing population of aging patients with multiple comorbidities in developing countries. As one of the most serious complications after cardiac surgery, the incidence of cardiac surgery-associated acute kidney injury (CSA-AKI) is reported ranging from 26.0- 
$28.5 \%$, supported by a recent meta-analysis [1]. CSA-AKI not only substantially affects acute morbidity and mortality, but also is strongly associated with increased risks of cardiovascular events, advanced chronic kidney disease, and long-term death [2-4].

Early identification of patients at high risk of AKI is essential in order to trigger the use of preventive care actions. In the past few decades, multiple efforts have been made to develop various models to predict AKI after cardiac surgery. The Mehta score [5], Simplified Renal Index (SRI) score [6], and Cleveland Clinic score [7] are most widely used scoring systems for predicting CSA-AKI in Western countries. Their predictive ability had been adequately validated in Caucasians. However, adoption of these models to Chinese patient population would be of challenge, since the demographic, clinical characteristics, and health care system are quite different from the existing model derivation cohorts.

In 2013 , the International Society of Nephrology promoted the " 0 by 25 " initiative for AKI, namely, zero deaths of patients with untreated acute kidney failure by 2025 [8]. The vision aims to improve the diagnosis and treatment of $\mathrm{AKI}$ and appears inspiring. In contrast to the United States, over the past decade, the proportion of cardiac surgery continues to rise in China $[9,10]$. However, to date, a little progress has been made in predicting CSA-AKI in Chinese cardiac patients. Besides, no model has been used to evaluate the subsequent clinical outcomes of CSA-AKI. Our first objective was therefore to establish a nomogram model, a simple risk-calculated tool, for CSA-AKI which included preoperative, intraoperative, and early postoperative variables with the data retrieving from three registers in China. The nomogram was then compared with that of five conventional models (Mehta score, $\mathrm{Ng}$ score [11], AKICS score [12], SRI score, and Cleveland Clinic score) to determine whether these models could be used to predict CSA-AKI in Chinese patients. Given that AKI acted as a powerful predictor of poor clinical prognosis, finally, we aimed to use the nomogram to evaluate mid-term outcomes of 7-year follow-up.

\section{Patients And Methods}

\section{Study design and participants}

Consecutive patients who had cardiac surgery at 14 institutes, China, admitted between January 2013 and December 2020, were recruited from three population-based registers: Jiangsu Province Coronary Artery Bypass Grafting Registry, Patient Data Management Platform, and Chinese Cardiac Surgery Registry. All data were de-identified and were pre-processed by the administrators at each institute. Before delivering for analysis, the data integrity was screened and examined by two administrators of each institution.

We enrolled patients who had received coronary artery bypass grafting surgery (CABG), valve surgery, and a combination of both treatments. Patients were excluded after meeting the following criteria: (i) younger than 18 years old; (ii) preoperative AKI, dialysis, or end-stage renal disease; (iii) kidney transplantation; (iv) missing data of serum creatinine. The research was approved by the Ethical Committee of Nanjing First Hospital, and patient informed consent was waived given its retrospective nature. 


\section{End Point Definition}

The primary end point was any-stage AKI with the definition of Kidney Disease Improving Global Outcomes (KDIGO) criteria [13], specifically, an increase in serum creatinine (Scr) from baseline of $\geq 0.3$ $\mathrm{mg} / \mathrm{dl}$ within 48 hours or $>1.5$ times the baseline level within 7 days, or a new requirement for renal replacement therapy. The Scr level measured before surgery was used as the reference value. We did not use urine output to define AKI because of its unavailability for the majority of patients. Secondary end points were 7-year follow-up outcomes: (i) death from all causes; and (ii) major adverse kidney events (MAKEs), defined as the composite outcome of death from renal failure, dialysis, and advanced chronic kidney disease (estimate glomerular filtration rate [eGFR] $<30 \mathrm{~mL} / \mathrm{min} / 1.73 \mathrm{~m}^{2}$ for at least three months). eGFR was calculated using the Chronic Kidney Disease Epidemiology Collaboration (CKD-EPI) creatinine equation [14].

\section{Potential Predictive Variables And Definitions}

The potential predictive variables are listed in Table 1. Quantitative variables were categorized according to the clinically meaningful cut-off values or previous literature reports. For example, we classified age into three groups, including $<60,60-70$, and $>70$ years. Scr was classified into three groups, including $<1.2$, 1.2-2.0, and $>2.0 \mathrm{mg} / \mathrm{dl}$. Cardiopulmonary bypass (CPB) time was dichotomized at a value of 116 minutes according to the maximal Youden index [15]. To compare with previous data $[12,16]$, we chose a cut-off value of 120 minutes for subsequent analysis. Renal disease without dialysis was defined as any one of the following renal diseases: hypertensive renal disease, diabetic nephropathy, primary glomerulonephritis, nephrotic syndrome, or chronic kidney disease with eGFR $<60 \mathrm{~mL} / \mathrm{min} / 1.73 \mathrm{~m}^{2}$, lasting more than three months. Critical preoperative state was defined as any one of the following preoperative situations: cardiogenic shock, cardiopulmonary resuscitation, ventricular fibrillation or flutter, intra-aortic balloon pump implantation, or an ejection fraction $<30 \%$. Other variables definitions are provided in Additional file, Table S1. This study adhered to the Transparent Reporting of a Multivariable Prediction Model for Individual Prognosis or Diagnosis (TRIPOD) guideline for reporting [17]. 
Table 1

Variable overview

\begin{tabular}{|ll|}
\hline Item & Variables \\
\hline $\begin{array}{l}\text { Patient } \\
\text { information } \\
\text { (six } \\
\text { variables) }\end{array}$ & Age, male, body mass index, center location, rural area, non-insurance \\
$\begin{array}{l}\text { Medical } \\
\text { history and } \\
\text { comorbidities } \\
\text { (25 variables) }\end{array}$ & $\begin{array}{l}\text { Smoker, diabetes mellitus, hyperlipidemia, hypertension, renal disease without } \\
\text { dialysis, peripheral vascular disease, chronic obstructive pulmonary disease, pleural } \\
\text { angina, cerebrovascular accident, critical preoperative state, infective endocarditis, } \\
\text { fibrillation, prior percutaneous coronary intervention, serum creatinine, hepatic } \\
\text { insufficiency, carotid stenosis, pulmonary hypertension, moderate to severe valve }\end{array}$ \\
$\begin{array}{ll}\text { insufficiency (aortic valve, mitral valve, and tricuspid valve), number of diseased } \\
\text { coronary vessels, left main disease }\end{array}$ \\
$\begin{array}{l}\text { (seven } \\
\text { variables) }\end{array}$ & $\begin{array}{l}\text { Nitrates, dopamine, metoprolol, angiotensin-converting enzyme inhibitor, statins, } \\
\text { aspirin, clopidogrel }\end{array}$ \\
$\begin{array}{l}\text { Procedure- } \\
\text { related } \\
\text { factors (five } \\
\text { variables) }\end{array}$ & $\begin{array}{l}\text { Previous open cardiac surgery, non-elective surgery, minimally invasive approach, } \\
\text { surgery type, surgeon experience. }\end{array}$ \\
$\begin{array}{l}\text { Intraoperative } \\
\text { and early } \\
\text { operative } \\
\text { factors (11 } \\
\text { variables) }\end{array}$ & $\begin{array}{l}\text { Cardiopulmonary bypass time, cardioplegic solution, perfusion strategy (antegrade, } \\
\text { intermittent), intra-aortic balloon pump, intraoperative transfusion (red blood cell, } \\
\text { plasma, cryoprecipitate, platelet), prolonged mechanical ventilation, reoperation for } \\
\text { bleeding }\end{array}$ \\
\hline
\end{tabular}

\section{Statistical analysis}

Categorical variables are presented as frequency (n) and proportion (\%). Quantitative variables are summarized with means \pm standard deviations in the case of normal distribution or medians (interquartile range) otherwise. Statistical differences between derivation and validation cohorts were carried out using the chi-square test, Fisher's exact probability method, t-test, or Mann-Whitney U-test as appropriate. As most data were collected through manual review of the medical records, missing values are unavoidable. Our dataset was highly complete with most variables having missing rates ranging 0 $4.1 \%$. The missing values were handled with multiple imputation method (mice package of $\mathrm{R}$, version 3.13.0).

The prediction model was developed in the derivation dataset and it consisted of two main stages: (i) feature selection; and (ii) multivariate logistic regression analysis. This is a logistic regression model. Multicollinearity of the variables was tested and excluded by establishing the variance inflation factor with maximum value set at 5 . To identify the risk factors, clinical characteristics ascertained during hospitalization were entered into the feature selection process using least absolute shrinkage and selection operator (LASSO). As a machine learning approach, LASSO regression adds the L1 
regularization as a penalty, shrinking the regression coefficients of weak factors towards zero with larger penalties [18]. It generates a model that minimizes the prediction error. The most predictive variables were determined by the tuning parameter (minimum lambda plus 1 standard error) using an automated 10 -fold cross-validation method (glmnet package of R, version 4.0-2).

Selected predictors were incorporated in a logistic regression analysis to generate a nomogram model ( $r m s$ package of $\mathrm{R}$, version 6.1-0). Model discrimination was assessed using the $\mathrm{C}$-statistic, an index equivalent to the area under the receiver operating characteristic curve (AUC). The relationship between predicted probabilities and observed outcome frequencies was accessed by the Brier score and was visualized with a calibration plot using 1000 bootstrap resampling method. Lower Brier scores indicate superior model calibration [19]. In both derivation and validation cohorts, we compared the discrimination and calibration of the nomogram with five conventional scoring systems: Mehta score, Ng score, AKICS score, SRI score, and Cleveland Clinic score. The clinical practice and benefits of the models were estimated by decision curve analyses [20] ( $r$ mda package of $R$, version 1.6). To facilitate clinical use of the model, we calculated the total score for each patient by summing the points of each factor. Patients were stratified into three risk groups based on the score distribution: low-risk group (<20th percentile), moderate-risk group (20th-80th percentile), and high-risk group (>80th percentile).

Next, we evaluated mid-term outcomes (two events: death from all causes and MAKEs) according to the three risk-stratified groups. Time-to-event analyses were performed using Cox proportional hazards models to compare death from all causes. Competing risk analysis was performed in the Fine and Gray model to compare MAKEs after adjusting death as a competing risk (cmprsk package of R, version 3.5.3). Hazard ratios (HRs), sub-distribution HRs (SHRs), and their two-sided 95\% confidence intervals (Cls) were determined. Statistical analyses were performed using R (version 4.0.3, http://www.r-project.org/). A twosided $P$ value $<0.05$ indicated statistically significant.

\section{Results}

\section{Characteristics of the cohorts}

From January 2013 to December 2020, 11740 participates met the inclusion criteria and were enrolled for analysis. They were randomly assigned to a derivation cohort $(n=8197)$ or a validation cohort $(n=$ 3543), with a split ratio of 7:3. The analysis workflow is shown in Additional file, Fig. S1. The baseline characteristics were similar between the two cohorts. The rates of AKI were $27.6 \%, 27.5 \%$, and $27.8 \%$ in the total, derivation, and validation cohorts, respectively (see Additional file, Table S2).

\section{Feature Selection}

54 variables during hospitalization were included in LASSO regression (Fig. 1A). The results showed that 14 variables remained significant predictors of AKI, including age, male, diabetes mellitus, hypertension, renal disease without dialysis, critical preoperative state, infective endocarditis, Scr, surgery type, CPB 
time, intra-aortic balloon pump, intraoperative red blood cell (RBC) transfusion, prolonged mechanical ventilation (PMV), and reoperation for bleeding (Fig. 1B). Inclusion of these 14 predictors in a multivariate logistic regression model resulted in 12 predictors (excluded male and reoperation for bleeding) that were statistically significant for AKI and were included in the final model (Table 2). 
Table 2

Multivariate logistic regression model showing the independent risk factors of acute kidney injury in the derivation cohort

\begin{tabular}{|c|c|c|c|}
\hline Factors & $\boldsymbol{\beta}$ & OR $(95 \% \mathrm{Cl})$ & $P$ value \\
\hline \multicolumn{4}{|l|}{ Age, years } \\
\hline $60-69$ vs $<60$ & 0.5909 & $1.81(1.56-2.09)$ & $<0.001$ \\
\hline$\geq 70$ vs $<60$ & 1.0776 & $2.94(2.50-3.46)$ & $<0.001$ \\
\hline \multicolumn{4}{|l|}{ Diabetes mellitus } \\
\hline NIDDM vs no history & 0.7228 & $2.06(1.75-2.42)$ & $<0.001$ \\
\hline IDDM vs no history & 1.2964 & $3.66(2.85-4.69)$ & $<0.001$ \\
\hline Hypertension, yes vs no & 0.5547 & $1.74(1.54-1.98)$ & $<0.001$ \\
\hline Renal disease without dialysis, yes vs no & 2.3274 & $10.25(6.59-15.94)$ & $<0.001$ \\
\hline Critical preoperative state, yes vs no & 1.1632 & $3.20(2.39-4.28)$ & $<0.001$ \\
\hline Infective endocarditis, yes vs no & 1.0847 & $2.96(2.28-3.84)$ & $<0.001$ \\
\hline \multicolumn{4}{|l|}{ Serum creatinine, $\mathrm{mg} / \mathrm{dl}$} \\
\hline $1.2-2.0$ vs $<1.2$ & 0.7530 & $2.12(1.74-2.60)$ & $<0.001$ \\
\hline$>2.0$ vs $<1.2$ & 2.2334 & $9.33(4.80-18.14)$ & $<0.001$ \\
\hline \multicolumn{4}{|l|}{ Surgery type } \\
\hline Valve surgery alone vs $C A B G$ alone & 0.6850 & $1.98(1.69-2.34)$ & $<0.001$ \\
\hline Combined surgery vs CABG alone & 1.5972 & $4.94(4.06-6.00)$ & $<0.001$ \\
\hline \multicolumn{4}{|l|}{ Cardiopulmonary bypass time, min } \\
\hline $1-120$ vs none & 0.7237 & $2.06(1.66-2.57)$ & $<0.001$ \\
\hline$>120$ vs none & 1.3248 & $3.76(2.95-4.79)$ & $<0.001$ \\
\hline Intra-aortic balloon pump, yes vs no & 1.7045 & $5.50(3.31-9.14)$ & $<0.001$ \\
\hline Intraoperative red blood cell transfusion, yes vs no & 0.9814 & $2.67(2.27-3.13)$ & $<0.001$ \\
\hline Prolonged mechanical ventilation, yes vs no & 0.7556 & $2.13(1.82-2.48)$ & $<0.001$ \\
\hline Intercept & -4.0269 & & \\
\hline
\end{tabular}

\section{Nomogram And Model Performance}


A nomogram model was generated based on the coefficients of the logistic regression (Fig. 2). The nomogram demonstrated good discrimination for predicting AKI, with a C-statistic of $0.825(95 \% \mathrm{Cl}$ $0.815-0.835$ ) and an adjusted C-statistic of 0.819 by internal validation. Correspondingly, in the validation cohort, the nomogram displayed a C-statistic of 0.804 (95\% Cl 0.789-0.820). The 1000-resample bootstrapped calibration plot showed an optimal agreement between the predicted risk and observed frequency in both cohorts (Fig. 3).

\section{Model Comparisons}

The performance of the nomogram model was compared with five conventional models: Mehta score, $\mathrm{Ng}$ score, AKICS score, SRI score, and Cleveland Clinic score. In both cohorts, the nomogram model had better discrimination and calibration than other five models. Decision curve analyses revealed that the nomogram model had a superior net benefit over the other five models for most of the examined probabilities (Additional file, Fig. S2).

\section{Risk Stratification And Mid-term Outcomes Evaluation}

In the derivation cohort, the patients were stratified into low-risk, moderate-risk, and high-risk groups with two knots score being placed at 60 and 165, corresponding to the 20th and 80th percentile of score distribution, respectively. The risks of AKI for the three risk groups were approximately similar in both cohorts (Additional file, Fig. S3).

The mid-term follow-up data were extracted from our center, and 2786 consecutive patients were available for analysis (January 2013-June 2019). These patients were allocated to low-risk group (score $<60, n=557$ ), moderate-risk group (score $\geq 60 / \leq 165, n=1625$ ), and high-risk group (score $>165, n=$ 604) by calculating each individual score. Data were censored on 31st January 2020. 149 patients (5.3\%) were lost to follow-up and were included in the analysis according to the last data recorded in the registry. The missing follow-up rates were $4.7 \%, 5.4 \%$, and $5.8 \%$ for low-risk, moderate-risk, and high-risk groups, respectively; no significant difference was found between the three groups $(P=0.683)$. Higher score group was associated with significantly higher probabilities of death and MAKEs during the 7-year followup. With the low-risk group used as the reference category, the rates of death from all causes were $8.9 \%$ (95\% Cl 6.3-11.3\%) for moderate-risk group (HR 2.01, 95\% Cl 1.12-3.61; $\mathrm{P}=0.012)$ and $19.4 \%(95 \% \mathrm{Cl}$ 12.9-25.5\%) for high-risk group (HR 4.50, 95\% $\mathrm{Cl}$ 2.47-8.22; $\mathrm{P}<0.001$ ) (Table 3 and Fig. 4A). Similar findings were observed for MAKEs after adjusting for death as a competing risk. The rates of MAKEs were 5.7\% (95\% Cl 3.1-9.5\%) for moderate-risk group (SHR 5.24, 95\% Cl 1.63-16.90; $\mathrm{P}=0.006$ ) and 10.2\% (95\% Cl 6.0-15.7\%) for high-risk group (SHR 11.30, 95\% Cl 3.47-36.71; $\mathrm{P}<0.001$ ) (Table 3 and Fig. 4B). 
Table 3

7-year cumulative incidences of death from all causes and MAKEs by risk groups

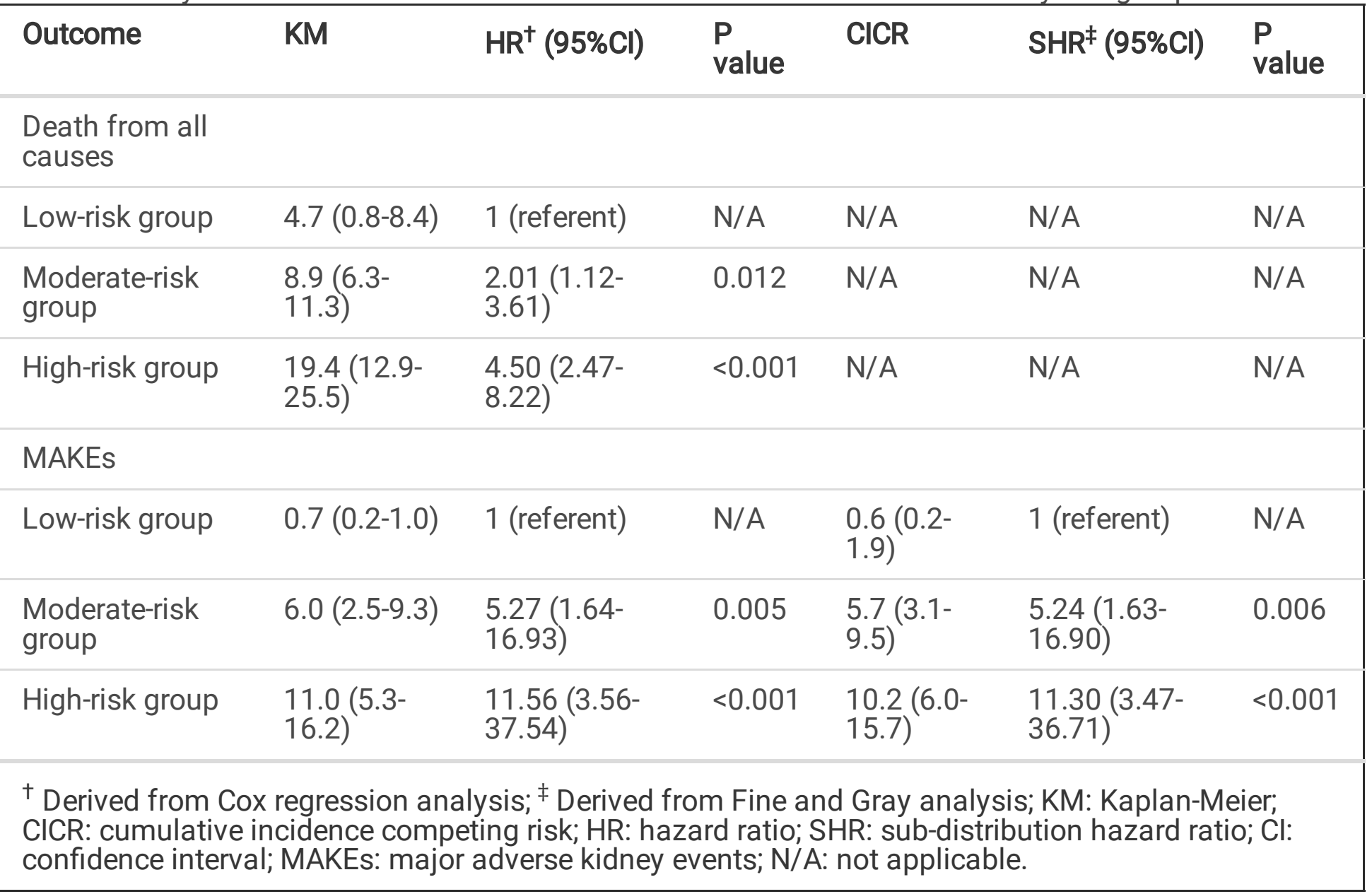

\section{Discussion}

Using a large cohort from 14 cardiac centers, we developed and validated a nomogram model to predict AKI and evaluate subsequent adverse outcomes for patients undergoing cardiac surgery in China. The nomogram demonstrated adequate discrimination and calibration in both cohorts and showed a better performance when compared with other five scoring systems. Additionally, we stratified patients into lowrisk, moderate-risk, and high-risk groups according to the score generated from the nomogram. Higher score group was associated with higher risks of death from all causes and MAKEs during 7-year followup. These findings suggested that the nomogram could serve as a risk-calculated tool for enhancing the risk stratification of CSA-AKI and its relevant clinical outcomes in Chinese cardiac patients.

Among 54 exposure variables, we identified 12 important risk factors associated with CSA-AKI. Variable selection procedure is one of the most important processes when constructing a prediction model. Logistic regression is commonly used. Traditional approach that included a set of variables to generate a model tends to lead to overfitting [21]. The feature selection strategies we applied in this study, which is a combination of machine learning and logistic regression method, provide insights on handing clinical data. LASSO is an alternative and effective option in handling high-dimensionality data. It penalizes magnitude of regression coefficients and excludes variables with a zero coefficient. A particular 
advantage of this technique is that it avoids both overfitting and overestimation during model derivation [18]. After this selection process, the strong predictors were identified in the final model. Several predictors identified in this study are consistent with previous researches, including age, diabetes mellitus, hypertension, critical preoperative state, Scr, surgery type, CPB time, and intraoperative RBC transfusion. Renal disease, infective endocarditis and PMV are new risk factors that have not been incorporated into those scoring systems. This indicated that, despite differing in races, AKI may have some common risk factors. However, these risk factors have different coefficients between races, and thus contributing different weights in the models.

We compared the performance of the five conventional models and found none of them demonstrated adequate power for predicting AKI in Chinese cardiac patients, both in terms of discrimination and calibration. Several factors may affect their wide application. First, the AKI definitions were various in the previous studies. In 2012, diagnostic criteria of AKI were revised significantly by the KDIGO organization. Therefore, the models established before 2012 may be imprecise and should be cautiously applied to current clinical practice [22]. Second, the Mehta score, Cleveland Clinic score, and SRI score were developed principally for AKI requiring renal replacement therapy (RRT-AKI), which is rare and not less severe stages of AKI, with incidences of $1.4-2.2 \%$ in their reports, obviously lower than the any-stage AKI rate (27.6\%) in the present study. Ranucci et al. [23] validated the three models in a single-center study. They found that the three models showed excellent predictive ability for RRT-AKI, but were not wellperformed for predicting non-RRT-AKI. Similarly, Che et al. [24] found that the Cleveland Clinic score and SRI score had poor classification (AUCs ranged from 0.516 to 0.673 ), and cannot be applied effectively in Chinese AKI patients. Third, most of previous prediction models only attached importance to preoperative variables. Given that the occurrence of AKI is a dynamic process and is particularly affected by procedure-related factors, we suggest that all preoperative, intraoperative, and early postoperative parameters should be screened for assessment during model derivation. Taken together, these data indicated that the study end point, race, and sample source are still pivotal factors and different models may be more suitable to apply to their specific populations.

Although AKI may be reversible, some of the patients develop mild or even transient AKI that could lead to CKD or adverse clinical outcomes $[25,26]$. Particularly in patients with pre-existing renal disease (e.g., in older patients, high levels of Scr), AKI substantially accelerates the severity of kidney dysfunction and its progression to end-stage renal disease or adverse events $[27,28]$. However, the pathophysiology and precise mechanism of AKI-to-CKD transition are complex and remain not fully understood. Notably, the nomogram model did not only predict CSA-AKI, higher score group was also associated with higher rates of mid-term death and MAKEs. These findings highlight the nomogram as a useful tool assisting in the risk stratification of $\mathrm{AKI}$ and mid-term outcomes. When considering the clinical implications, we suggest that this model may be useful in enriching patient cohorts for clinical trials or establishing benchmarks of cardiac surgical care. Using the nomogram may help in choosing preventive strategies in the perioperative management of patients. These strategies might include individualized blood pressure control, change of the surgical procedures (e.g., change from on-pump to off-pump surgery), reduced CPB time, and intensified hemodynamic monitoring and airway management (e.g., early extubation). 
Our study has several strengths. We included 14 hospitals, and more than 11000 Chinese cardiac patients. Compared with previous study of AKI, defined as RRT-AKI, a definition of any-stage AKI was used in this study, thus extending the risk model to milder AKI patients. Besides, our study revealed that AKI is strongly associated with poor clinical prognosis; an AKI prediction model could also be useful for evaluating subsequent adverse outcomes of CSA-AKI. However, several limitations of this study should also be acknowledged. First, although the data were prospectively collected with adequate quality, the ascertainment or selection bias from a retrospective in design could not be fully avoided. Second, the model was developed based on routine variables extracted from the electronic health records. Therefore, the risk model can only apply the variables that have been collected. Third, although we developed and validated the model with a multicenter data resource, the model was not externally validated in other races or regions. It would be of caution to apply the model to other developing nations. External validation of the model in larger scale of Chinese patient cohorts is also needed.

\section{Conclusion}

The nomogram provided an effective tool for predicting acute kidney injury and evaluating its subsequent adverse events after cardiac surgery.

\section{Abbreviations}

CSA-AKI: cardiac surgery-associated acute kidney injury; CABG: coronary artery bypass grafting surgery; KDIGO: Kidney Disease Improving Global Outcomes; Scr: serum creatinine; MAKEs: major adverse kidney events; eGFR: estimate glomerular filtration rate; CPB: cardiopulmonary bypass; PMV: prolonged mechanical ventilation; LASSO: least absolute shrinkage and selection operator; AUC: area under the receiver operating characteristic curve; HR: hazard ratio; SHR: sub-distribution hazard ratio; Cl: confidence interval.

\section{Declarations}

\section{Ethics approval and consent to participate}

The Ethical Committee of Nanjing First Hospital approved the study. Consent to participate for this study was waived because no individual patients were identified.

\section{Consent for publication}

Not applicable.

\section{Availability of data and materials}

All data were retrieved from the databases and are available from the corresponding author on reasonable request. 


\section{Competing interests}

None declared.

\section{Funding}

This work was supported by the National Natural Science Foundation of China (No. 8217021245 to Xin Chen), Clinical Research Plan of SHDC (No. SHDC2020CR3100B to Min Yu), and Changzhou Science and Technology Support Program (Social Development) (No. CE20205047 to Ke Zhang).

\section{Authors' contributions}

We confirm that the manuscript has been read and approved by all named authors. We further confirm that the order of authors listed in the manuscript has been approved by all of us. HZ, MY participated in conception and design of the study. HZ, RW, RF, and KZ obtained, organized and cleaned the dataset. $\mathrm{HZ}$, MY performed data analyses. HZ, WC, and XC drafted the article.

\section{Acknowledgements}

None.

\section{Author details}

${ }^{1}$ Department of Thoracic and Cardiovascular Surgery, Nanjing First Hospital, Nanjing Medical University, No. 68 Changle Road, Nanjing 210006, China. ${ }^{2}$ Department of Cardiovascular Surgery, Shanghai General Hospital, Shanghai Jiao Tong University School of Medicine, No. 100 Haining Road, Shanghai 200080, China. ${ }^{3}$ Department of Thoracic and Cardiovascular Surgery, Changzhou Second People's Hospital, Nanjing Medical University, No. 68 Gehu Middle Road, Changzhou 213003, China.

\section{References}

1. Neugarten J, Sandilya S, Singh B, Golestaneh L. Sex and the Risk of AKI Following Cardio-thoracic Surgery: A Meta-Analysis. Clin J Am Soc Nephrol. 2016;11(12):2113-22. doi:10.2215/cjn.03340316.

2. James MT, Bhatt M, Pannu N, Tonelli M. Long-term outcomes of acute kidney injury and strategies for improved care. Nat Rev Nephrol. 2020;16(4):193-205. doi:10.1038/s41581-019-0247-z.

3. Hobson CE, Yavas S, Segal MS, Schold JD, Tribble CG, Layon AJ, et al. Acute kidney injury is associated with increased long-term mortality after cardiothoracic surgery. Circulation. 2009;119(18):2444-53. doi:10.1161/circulationaha.108.800011.

4. Vanmassenhove J, Kielstein J, Jörres A, Biesen WV. Management of patients at risk of acute kidney injury. Lancet. 2017;389(10084):2139-51. doi:10.1016/s0140-6736(17)31329-6.

5. Mehta RH, Grab JD, O'Brien SM, Bridges CR, Gammie JS, Haan CK, et al. Bedside tool for predicting the risk of postoperative dialysis in patients undergoing cardiac surgery. Circulation. 
2006;114(21):2208-16. doi:10.1161/circulationaha.106.635573.

6. Wijeysundera DN, Karkouti K, Dupuis JY, Rao V, Chan CT, Granton JT, et al. Derivation and validation of a simplified predictive index for renal replacement therapy after cardiac surgery. JAMA. 2007;297(16):1801-9. doi:10.1001/jama.297.16.1801.

7. Thakar CV, Arrigain S, Worley S, Yared JP, Paganini EP. A clinical score to predict acute renal failure after cardiac surgery. J Am Soc Nephrol. 2005;16(1):162-8. doi:10.1681/asn.2004040331.

8. Remuzzi G, Horton R. Acute renal failure: an unacceptable death sentence globally. Lancet. 2013;382(9910):2041-2. doi:10.1016/s0140-6736(13)62193-5.

9. Bowdish ME, D'Agostino RS, Thourani VH, Desai N, Shahian DM, Fernandez FG, et al. The Society of Thoracic Surgeons Adult Cardiac Surgery Database: 2020 Update on Outcomes and Research. Ann Thorac Surg. 2020;109(6):1646-55. doi:10.1016/j.athoracsur.2020.03.003.

10. Zheng Z, Zhang H, Yuan X, Rao C, Zhao Y, Wang Y, et al. Comparing Outcomes of Coronary Artery Bypass Grafting Among Large Teaching and Urban Hospitals in China and the United States. Circ Cardiovasc Qual Outcomes. 2017;10(6):e003327. doi:10.1161/circoutcomes.116.003327.

11. Ng SY, Sanagou M, Wolfe R, Cochrane A, Smith JA, Reid CM. Prediction of acute kidney injury within 30 days of cardiac surgery. J Thorac Cardiovasc Surg. 2014;147(6):1875-83. 83.e1

. doi:10.1016/j.jtcvs.2013.06.049.

12. Palomba H, de Castro I, Neto AL, Lage S, Yu L. Acute kidney injury prediction following elective cardiac surgery: AKICS Score. Kidney Int. 2007;72(5):624-31. doi:10.1038/sj.ki.5002419.

13. Inker LA, Astor BC, Fox CH, Isakova T, Lash JP, Peralta CA, et al. KDOQI US commentary on the 2012 KDIGO clinical practice guideline for the evaluation and management of CKD. Am J Kidney Dis. 2014;63(5):713-35. doi:10.1053/j.ajkd.2014.01.416.

14. Levey AS, Stevens LA, Schmid CH, Zhang YL, Castro AF 3rd, Feldman HI, et al. A new equation to estimate glomerular filtration rate. Ann Intern Med. 2009;150(9):604-12. doi:10.7326/0003-4819150-9-200905050-00006.

15. Fluss R, Faraggi D, Reiser B. Estimation of the Youden Index and its associated cutoff point. Biom J. 2005;47(4):458-72. doi:10.1002/bimj.200410135.

16. Nah CW, Ti LK, Liu W, Ng RR, Shen L, Chew ST. A clinical score to predict acute kidney injury after cardiac surgery in a Southeast-Asian population. Interact Cardiovasc Thorac Surg. 2016;23(5):75761. doi:10.1093/icvts/ivw227.

17. Collins GS, Reitsma JB, Altman DG, Moons KG. Transparent reporting of a multivariable prediction model for individual prognosis or diagnosis (TRIPOD): the TRIPOD statement. BMJ. 2015;350:g7594. doi:10.1136/bmj.g7594.

18. Ranstam J, Cook JA. LASSO regression. Br J Surg. 2018;105(10):1348. doi:10.1002/bjs.10895.

19. Cook NR. Statistical evaluation of prognostic versus diagnostic models: beyond the ROC curve. Clin Chem. 2008;54(1):17-23. doi:10.1373/clinchem.2007.096529. 
20. Vickers AJ, Elkin EB. Decision curve analysis: a novel method for evaluating prediction models. Med Decis Making. 2006;26(6):565-74. doi:10.1177/0272989x06295361.

21. Ranstam J, Cook JA. Statistical models: an overview. Br J Surg. 2016;103(8):1047. doi:10.1002/bjs.10240.

22. Stevens PE, Levin A. Evaluation and management of chronic kidney disease: synopsis of the kidney disease: improving global outcomes 2012 clinical practice guideline. Ann Intern Med. 2013;158(11):825-30. doi:10.7326/0003-4819-158-11-201306040-00007.

23. Ranucci M, Aloisio T, Cazzaniga A, Di Dedda U, Gallazzi C, Pistuddi V. Validation of renal-risk models for the prediction of non-renal replacement therapy cardiac surgery-associated acute kidney injury. Int J Cardiol. 2018;272:49-53. doi:10.1016/j.ijcard.2018.07.114.

24. Che M, Wang X, Liu S, Xie B, Xue S, Yan Y, et al. A Clinical Score to Predict Severe Acute Kidney Injury in Chinese Patients after Cardiac Surgery. Nephron. 2019;142(4):291-300. doi:10.1159/000499345.

25. Liotta M, Olsson D, Sartipy U, Holzmann MJ. Minimal changes in postoperative creatinine values and early and late mortality and cardiovascular events after coronary artery bypass grafting. Am J Cardiol. 2014;113(1):70-5. doi:10.1016/j.amjcard.2013.09.012.

26. Mehta RL, Pascual MT, Soroko S, Savage BR, Himmelfarb J, Ikizler TA, et al. Spectrum of acute renal failure in the intensive care unit: the PICARD experience. Kidney Int. 2004;66(4):1613-21. doi:10.1111/j.1523-1755.2004.00927.x.

27. Ishani A, Xue JL, Himmelfarb J, Eggers PW, Kimmel PL, Molitoris BA, et al. Acute kidney injury increases risk of ESRD among elderly. J Am Soc Nephrol. 2009;20(1):223-8. doi:10.1681/asn.2007080837.

28. James MT, Pannu N, Hemmelgarn BR, Austin PC, Tan Z, McArthur E, et al. Derivation and External Validation of Prediction Models for Advanced Chronic Kidney Disease Following Acute Kidney Injury. JAMA. 2017;318(18):1787-97. doi:10.1001/jama.2017.16326.

\section{Figures}

\section{Figure 1}

Feature selection using least absolute shrinkage and selection operator regression. (A) Coefficient profiles of 54 variables. (B) Identification of optimal penalization coefficient using 10-fold cross-validation via minimum lambda plus 1 standard error criterion (14 variables). 


\section{Figure 2}

Nomogram to predict the probability of cardiac surgery-associated acute kidney injury. NIDDM: noninsulin-dependent diabetes mellitus; CABG: coronary artery bypass grafting; CPB: cardiopulmonary bypass; PMV: prolonged mechanical ventilation; Cl: confidence interval. 


\section{Figure 3}

Model performance for evaluating cardiac surgery-associated acute kidney injury. The area under the curves of the nomogram for predicting acute kidney injury in the derivation (A) and validation (B) cohorts. Calibration curves of the nomogram for predicting acute kidney injury in the derivation (C) and validation (D) cohorts. AUC: area under the receiver operating characteristic curve; Cl: confidence interval.

\section{Figure 4}

Cumulative incidence curves for death from all causes (A) and major adverse kidney events (B) after risk stratification. MAKEs: major adverse kidney events.

\section{Supplementary Files}

This is a list of supplementary files associated with this preprint. Click to download.

- SupplementaryMaterial.docx 